\title{
MAKING A DIFFERENCE: THE INNOVATION POTENTIAL OF ADDITIVE MANUFACTURING
}

\author{
Shivohum Loyalka \\ Modern School Vasant Vihar, New Delhi \\ DOI: 10.46609/IJSSER.2021.v06i06.019 URL: https://doi.org/10.46609/IJSSER.2021.v06i06.019
}

\begin{abstract}
Additive manufacturing has allowed for businesses across the world to take advantage of reduced time taken to manufacture products, keep up with decreased lead times, and the challenges posed by online commerce. Automation has taken place in every aspect of the retail product development industry, and the rise of 3D printing has led to exponential industry and economic growth. However, research has shown that it is only the large manufacturers and corporations which have been able to take full advantage of additive manufacturing technologies, while small and medium sized enterprises are being left behind and face several challenges investing in such technologies and implementing them depending on their position in the supply chain. This paper will examine the economics behind 3D printing and additive manufacturing technologies, and emphasise the need for support to SMEs to achieve competitive advantages in using the technologies. Drawing from successful examples globally, the paper will pose policy recommendations for improved support to SMEs and guiding them through all the various stages required in implementing these technologies. The paper will also pose recommendations on reskilling and the requirement to train the existing workforce to meet the challenges of automation.
\end{abstract}

Keywords: Supply Chain, Enterprises, Manufacturing, technology, SMEs

\section{Introduction}

With technological improvements and increase in automation, there has been a rise in 3D printing and additive manufacturing technologies in India as well as other parts of the world. Automation will essentially reshape retail businesses and particularly in retail product development, creating better organisations with fewer layers along the value chain and a better trained and empowered workforce (McKinsey, 2019). Research has shown that about half of the activities in retail can be automated using current, at-scale technology, such as additive manufacturing and 3D printing (McKinsey, 2019). The current challenges posed to the market 


\section{International Journal of Social Science and Economic Research}

ISSN: $2455-8834$

Volume:06, Issue:06 "June 2021"

have led to a requirement for shorter product cycles and lead times and fulfilling better customization within the production lines (Shah and Mattiuza, 2018). Therefore, many productbased manufacturing enterprises have found that automation through additive manufacturing, which "adopts the process of joining materials through layers to produce objects through 3D digital data technology that enables it to innovate the traditional manufacturing processes" (Shah and Mattiuiza, 2018).

However, with the potential for innovation and automation comes the importance of analyzing economic viability of such technologies for different levels of the economy. Pursuing additive manufacturing can be especially challenging for small and medium sized enterprises (hereafter referred to as SMEs), and those enterprises which are a part of the supply chain of larger firms (Martinsuo and Luomaranta, 2018). It is crucial to understand the challenges and strategies for adoption of additive manufacturing for SMEs, in different positions along the supply chain, and adopt strategic and operative actions to combat those challenges and ensure equitable adoption of these technologies for maximum benefit and preventing loss of employment (Martinsuo and Luomaranta, 2018). This paper will examine the existing literature on automation and the merits and demerits of additive manufacturing, with focus on retail product development. This paper will evaluate the conceptual underpinnings of additive manufacturing and 3D printing technology and assess the economic viability of these technologies for SMEs. Examining the impacts and global examples, the paper will then pose policy recommendations for improving the accessibility of such technologies to SMEs and the implications of the same for the global economy.

\section{Background and Relevant Literature}

The field of retail product development has been under strain. Factors which are putting the industry under strain are higher costs to manage e-commerce supply chains, growing demands from suppliers to pass on raw-material cost inflation, higher investments to match new competition, and steadily rising labor costs (McKinsey, 2019). Customers demand faster turnaround due to the development of e-commerce and online shopping, which developed in the 1990s and the 2000s (Subha, 2019), automation and 3D printing technologies are playing a big role in scaling manufacturing industries. Research shows that the use cases for additive manufacturing have only increased over the years, from prototyping to now producing production parts, bridge production, repair or jigs, fixtures, and tooling (Leedale, 2020). 3D printing has now developed to produce parts for even the most demanding industrial application (Leedale, 2020).

The global market for 3D printing was estimated to be USD 2183 million in 2012, and is expected to reach USD 8675.7 million by 2020, growing at a CAGR of $18.9 \%$ from 2013 to 


\section{International Journal of Social Science and Economic Research}

ISSN: $2455-8834$

Volume:06, Issue:06 "June 2021"

2020 (Nikitakos et al, 2020). The economics of 3D printing comes with its own challenges. It is typically part of a three-step production process which also includes blasting or shot peening and a machining step, which means relying on two different types of machines to complete an entire part and scheduling human resources to do the machining. 3D printing also has a limited capacity, which requires planning to avoid volume bottlenecks, which involves strategic sourcing and often, integrating enterprise resource planning software (Leedale, 2020). The costs of 3D printing components and additive manufacturing technology, while reducing, are still high, which limits their adoption by SMEs (Willson and Woolin, 2018). Standards for AM produced products are only now being developed so manufacturing items for use in a regulated application, can be difficult to validate to the regulators' satisfaction (Willson and Woolin, 2018). Nikitakos et al, (2020) have characterized the market structure for additive manufacturing and 3D printing by four patterns: (1) Small production output, (2) high product complexity, (3) high demand for customization tailored to the individual needs of customers, (4) spatially remote demand for products and decentralized production of replacement parts.

In general, it is accepted that there is still a lack of research on the variables that affect the Return on Investment for a business adopting additive manufacturing. However, it is an emerging understanding that 3D printers are no longer considered to be stand alone machines, but as part of an end to end production workflow which includes material handling systems, multiple additive manufacturing systems, part handling systems, part cleaning systems, curing/heat treatment systems, support/powder removal and recycling systems, part inspection systems and part finishing systems (Molitch-Hou, 2018). In addition, it requires the integration of several components, namely (i) print properties selection, (ii) print preparation and orientation, (iii) managing in print queue, (iv) initiating printing, (v) removing parts from the 3D printer, (vi) post processing, and (vii) finished part retrieval (Greenfield, 2020).

Therefore, the direct transformation of three-dimensional data which is stored in a file to the end product, and obviating the need for the step of assembly provides efficiencies for products to be produced at scale (Nikitakos et al, 2020). However, it is clear that the integration of additive manufacturing and 3D printing involves significant cost as well as reworking the approach to automation, reskilling and training of the labour force, and managing end to end workflow. Given this, it is crucial to extend support to SMEs with regard to adopting and gaining benefits from these technologies. The following section of this paper will examine the aspect of economic viability and how additive manufacturing stands to impact the management of business, especially SMEs.

Discussion and Analysis 


\section{International Journal of Social Science and Economic Research}

ISSN: $2455-8834$

Volume:06, Issue:06 "June 2021"

It is clear that additive manufacturing and 3D printing has come with benefits, such as reducing production time, increasing efficiencies, and the ability to respond to rapidly changing customer demands for custom items or redesigns without the need for costly re-tooling, and the ability to minimize stock holding since items can be 'made to order' rather than held in a warehouse, and reducing waste during the manufacturing process (Wilson and Woolin, 2018). A report by the European Parliament notes that 3D printing could be beneficial for businesses by offering them various advantages (European Commission, n.d). These include decreasing the burden on logistical chains, lessening environmental impact, and reducing storage and transport operations and cost savings related to goods insurance. This shows that there is a clear case for economic viability (European Commission, n.d).

From a review of the literature as detailed above, additive manufacturing stands to impact operations management, scheduling, and factory floor management, as well as training of the labour force, etc. The adoption of additive manufacturing involves an evaluation of the following factors for effective integration: (1) strategic factors, such as business, technology, and manufacturing strategy, (2) organisational factors such as business size, organisational structure and culture, and workforce experience and skills, (3) operational factors such as product design, production planning/control, integration and cost accounting systems, and (4) supply chain factors such as customer and supply relations, vendor relations, logistics and distribution (Shah and Matiuzza, 2018). Therefore, it is key to make these technologies more accessible to SMEs, in order to take the most advantage for national and global economies.

Research has also identified that lack of financial resources and support is one of the key drawbacks towards investing in technological innovation and its adoption among SMEs. Some of the other drawbacks identified have been the lack of governmental policies, credit ratings, training and skills, market awareness and knowledge, skilled and experienced technical people (Shah and Matiuzza, 2018). There have been some successful examples globally, where 3D printing and additive manufacturing technology has been made more accessible to SMEs. For example, the EU found that while larger manufacturers were able to take advantage of additive manufacturing, while SMEs were left behind. The EU therefore started the AMable Project, to help SMEs across all stages to speed up the adoption of additive manufacturing technologies and assist with overcoming obstacles such as a lack of skilled human resources, access to know-how, equipment, infrastructure and markets. The range of additive manufacturing support services are provided through an open source and application programming interface-based digital marketplace, which will help the SMEs develop their ideas to a final product (European Commission, n.d). Another example is the support provided by the The Welding Institute (TWI) by way of a pilot project of free manufacturing and engineering support to SMEs. An example is an SME named Photocentric 3D, which was able to use the support and contacts with specialist 


\section{International Journal of Social Science and Economic Research}

ISSN: $2455-8834$

Volume:06, Issue:06 "June 2021"

suppliers of additive manufacturing support software to was able to carry out mechanical testing of parts created using its equipment, establishing performance figures it could use in its product information and marketing materials (TWI, n.d). Facilities such as the $3 \mathrm{M}$ Buckley Innovation Centre (3M BIC) in Huddersfield, in conjunction with the National Physical Laboratory (the UK's National Measurement Institute), have also been successful in providing holistic support to SMEs seeking to adopt additive manufacturing and developing products and solutions through 3D printing (Wilson and Woolin, 2018).

In addition, it is crucial that SME support not only include support and information about the benefits of adopting additive manufacturing technologies, but also be made aware of the risks of investing in such technology vis-à-vis outsourcing, possibilities of stress defects or deviation from the CAD software, and the requirement for enhancing the skillset of current members of staff who can undertake the process in-house, whether it is production or post-processing, through non-technical and technical certifications which are available (Wilson and Woolin, 2018).

\section{Conclusion}

It is clear the additive manufacturing can lead to exponential economic growth in the coming years. However, to harness the potential of additive manufacturing technologies will involve finding a balance between the benefits brought about by the technology, and economic interests of SMEs and their ability to fairly compete in the market. To this end, it is crucial for world governments to provide some form of institutional support to SMEs, allowing them to take advantage of the efficiencies that arise with adopting 3D printing and other AM technologies. It is also important to evaluate new and original paths of innovation that enterprises are adopting towards the implementation of additive manufacturing applications (Shah and Matuizza, 2018).

Further, SMEs must be encouraged and incentivized to invest in reskilling to cope with the maintenance and support for the end to end processes that will be required. Research shows that only about 5 percent of all jobs can be automated with the current technology, which provides an opportunities for companies (McKinsey, 2019), especially SMEs to realise that investing in reskilling can enable them to gain the maximum efficiencies of additive manufacturing technologies. Experts note that there will be an increase in jobs which is necessitated by distributed manufacturing, in which 3D printers will be located closer to the point of demand. For companies to succeed in the new paradigm of additive manufacturing, they will require more employees who can leverage local hubs of production near their customers and provide superior products to what can be sourced via a container ship (Molitch-Hou, 2018). SMEs further require assistance which is tailored to their position in the supply chain, which changes the focus for their adoption of 3D printing or other technologies. 
International Journal of Social Science and Economic Research

ISSN: 2455-8834

Volume:06, Issue:06 "June 2021"

As a concluding note, it is also important when considering any type of technological transformation to take into account the social and ethical consequences of adopting technologies at the expense of employment. While employment may be gained in particular areas, there may be losses in other areas to compensate, as well as a layoff of older workers who cannot meet the requirements of reskilling. Therefore, companies, SMEs, and governments must tread the path of adopting additive manufacturing carefully, balancing benefit with negative impact.

\section{References}

European Commission, (n.d), 'How to prepare SMEs for the uptake of additive manufacturing', https://cordis.europa.eu/article/id/125359-how-to-prepare-smes-for-the-uptake-of-additivemanufacturing

Greenfield, D, (2020), 'Automating Additive Manufacturing', Automation World, https://www.automationworld.com/factory/3d-printing-additive

manufacturing/article/21142717/automating-additive-

manufacturing\#: :text=To\%20optimize\%203D\%20printing\%20of,would\%20have\%20otherwise $\% 20$ been\%20unachievable.

Leedale, B, (2020), '3D Printing Meets Industrial Production', SME, https://www.sme.org/technologies/articles/2020/july/3d-printing-meets-industrial-production/

Martinuso, M, and Luomaranta, T, (2018), 'Adopting additive manufacturing in SMEs: exploring the challenges and solutions', Journal of Manufacturing Technology Management, Vol 29, Issue 4, 10.1108/JMTM-02-2018-0030

McKinsey and Company (2019), 'Automation in retail: An executive overview for getting ready', https://www.mckinsey.com/industries/retail/our-insights/automation-in-retail-an-executiveoverview-for-getting-ready

Molitch-Hou, M, (2018), 'Automated 3D Printing: How Industrial Additive Manufacturing Is Evolving', Engineering.com, https://www.engineering.com/story/automated-3d-printing-howindustrial-additive-manufacturing-is-evolving

Nikitakos, et al, (2020), 'Economics in 3D printing', Chapter 6 In 3D Printing: Applications in Medicine and Surgery, Elsevier Inc.

Shah, S, and Mattiuza, S, (2018), 'Adoption of Additive Manufacturing Approaches: The Case of Manufacturing SMEs', IEEE International Conference on Engineering, Technology and Innovation, https://ieeexplore.ieee.org/document/8436257 
International Journal of Social Science and Economic Research

ISSN: 2455-8834

Volume:06, Issue:06 "June 2021"

The Welding Institute, (n.d), 'Additive Manufacturing Support For Smes From Twi', https://www.twi-global.com/media-and-events/insights/additive-manufacturing-support-forsmes-from-twi

Wilson, M, and Woolin, R, (2018), 'How SMEs can adopt additive manufacturing', TCT Magazine, https://www.tctmagazine.com/tctblogs/guest-blogs/how-smes-can-adopt-additivemanufacturing/ 